\title{
CRECIMIENTO COMPARATIVO DE LA CONCHA DE ABANICO (Argopecten purpuratus ) EN SISTEMAS SUSPENDIDOS
}

\section{COMPARATIVE GROWTH OF PERUVIAN SCALLOP (Argopecten purpuratus ) IN SUSPENDED SYSTEMS}

\author{
Rosario Cisneros $^{1}$, Jorge Bautista ${ }^{1}$ y Juan Argüelles ${ }^{2}$
}

\section{Resumen}

Con el objetivo de determinar diferencias en el crecimiento de la concha de abanico Argopecten purpuratus procedentes de diferentes lugares, se realizaron dos experimentos de cultivo comparativo en sistemas suspendidos, tales como "pearl-nets" y linternas. El primer experimento se realizó con semillas obtenidas en laboratorio (IMARPE) y semillas silvestres colectadas en Bahía Independencia, Pisco; en el segundo, las semillas fueron colectadas de banco natural en Bahía Samanco y Lagunillas, Pisco. El trabajo se realizó en la playa "El Carbón", Pucusana. Para ambas experiencias se estimaron tasas de crecimiento, los parámetros $\mathrm{K}$ y L $\infty$, índice de crecimiento $(\Phi)$ y factor de condición $(F C)$. Se aplicó un análisis de covarianza (ANCOVA) y una prueba t para comparar las curvas de regresión longitud- peso.

Los resultados mostraron diferencias en el crecimiento y factor de condición entre los grupos estudiados. En todos los casos las tasas de crecimiento observadas son comparables con los estimados por otros autores. Se discute que las diferencias se deberían a la variabilidad genética entre las poblaciones de donde fueron obtenidas las semillas.

Palabras clave: cultivo, Argopecten purpuratus, parámetros, crecimiento, longitud

\begin{abstract}
In order to determine differences in growth of the Peruvian scallop Argopecten purpuratus from different areas, two comparative experiments in suspended systems, such as pearl-nets and lantern nets, were carried out. The first one, was performed with seeds obtained from the hatchery at IMARPE, and wild seeds collected at Independence Bay, Pisco; the second one, was done with wild seeds from Samanco Bay and Lagunillas, Pisco. The study was carried out in Playa "El Carbón", Pucusana. For both experiments growth rates, K and L $\infty$, parameters, growth rate $(\Phi)$ and condition factor, were estimated. An analysis of covariance (ANCOVA) and a t-test to compare the regression curves of the length-weight relationship were applied. The results showed differences in growth rates and condition factor, among the studied groups. In all cases the growth rates are comparable with values estimated by other authors. It is argued that differences estimated are due to a genetic variability among populations from different seed sources.
\end{abstract}

Key words: culture, Argopecten purpuratus, parameters, growth, length

\section{Introducción}

La producción global de pectínidos se ha incrementado a paso rápido en las últimas decadas, en el 2001 la industria produjo mas de 1.2 millones de toneladas métricas de pectínidos, aproximadamente 3.5 veces más que en 1990 (Uddin et al., 2007). Sin embargo el incremento de la demanda internacional, repercute con elevadas capturas de esta especie, en las principales zonas de extracción, declinando sus poblaciones (Moragat et al., 2001; Tarazona et al., 2007; Pacheco \& Gárate, 2005; Mendo \& Wolff, 2003).

La concha de abanico peruana Argopecten purpuratus (Lamarck 1819), es una de las doce especies de pectínidos que se comercializan en el mercado internacional, de alto valor nutritivo y gran aceptación en los Estados Unidos, Japón y Europa, particularmente Francia (De Franssu, 1990). Se distribuye en bahías someras desde Paita, Perú $\left(5^{\circ} \mathrm{S}, 81^{\circ} \mathrm{W}\right)$ a Coquimbo, Chile (Alamo \& Valdivieso, 1987), incluso puede llegar hasta Valparaiso, Chile $\left(33^{\circ} \mathrm{S}, 71^{\circ} \mathrm{W}\right)$ (Pacheco \& Stotz, 2006; Cantillanez et al., 2005). Es una de las principales especies de invertebrados marinos, que por decadas se ha extraido mediante pesca de buceo y desde 1982 se ha cultivado con éxito (Wolff, 1988). En el Perú, su población esta distribuida entre 5-40 $\mathrm{m}$ de profundidad a lo largo de toda la costa, pero los bancos naturales más grandes y las pesquerías estan concentradas en solo dos áreas : la Bahía de Sechura en el Norte y Bahía Independencia en el Sur (Wolff et al., 2007).

Se ha observado que los volúmenes de desembarque se incrementan durante eventos El Niño (1983/84 o 1997/98), cuyas condiciones favorecen a la 
especie (Mendo et al., 1988 ; Mendo \& Wolff, 2003); así mismo su abundancia fluctua grandemente, debido a las variaciones en el reclutamiento durante este evento (Pacheco \& Stotz, 2006).

El cultivo de concha de abanico depende fundamentalmente del abastecimiento de semillas, que tiene actualmente dos fuentes principales: la producción en ecloserías y la captación en el ambiente natural (Bandin \& Mendo, 1999). Aunque en los últimos años, en el país se ha desarrollado en base a semilla extraida del ambiente natural. Esto ha ocasionado que la principal restricción para el desarrollo acuícola sea la una reducida disponibilidad de ejemplares adultos en las poblaciones silvestres (Mendo et al., 2001).

El crecimiento es uno de los aspectos esenciales para el conocimiento de la dinámica poblacional de un recurso en explotación como es el caso de la concha de abanico (Yamashiro \& Mendo, 1988), así mismo uno de los factores para establecer la rentabilidad del cultivo y su sostenibilidad en el tiempo, es determinar si semillas obtenidas en laboratorio y colectadas en banco natural presentan diferencias de crecimiento (debido a su variabilidad genética) durante su cultivo en el mar y si existen diferencias de crecimiento entre poblaciones procedentes de diferentes localidades, motivo por el cual se realizó la presente experiencia.

\section{Materiales y Métodos}

Area de estudio y obtención de semillas

Durante el periodo de Junio del 2000 a Diciembre del 2001, se realizaron 2 experimentos de cultivo con Argopecten purpuratus, en la Playa "El Carbón", Pucusana $\left(73^{\circ} 47^{\prime} 7.57^{\prime}\right.$ 'W y $12^{\circ} 26^{\prime} 58^{\prime}$ 'S $\left.\mathrm{S}\right)$, ubicada a $60 \mathrm{Km}$ al sur de la ciudad de Lima (Figura 4). El primer experimento se realizó de Junio a Noviembre del 2000, con semillas obtenidas en el laboratorio de Cultivos Marinos del Instituto del Mar del Perú (IMARPE), mediante inducción al desove de reproductores de concha de abanico procedentes de cultivo (Di Salvo et al., 1984; Cisneros et al., 1992); otro grupo fue colectado en Bahía Independencia, Pisco, principal centro de desembarque de concha de abanico en el litoral (Yamashiro et al., 2002; Mendo \& Wolff, 2003).

El segundo experimento se realizó de Junio a Diciembre del 2001, con semillas silvestres colectadas en diferentes bancos naturales, Bahía Samanco, Ancash y Lagunillas, Pisco.

$\underline{\text { Sistemas de cultivo y densidad de siembra }}$

Se instaló una línea de cultivo, ubicada a $5 \mathrm{~m}$ de la superficie del mar, en la que se colocaron sistemas de crianza. "Pearl-nets" para el cultivo intermedio y linternas para el cultivo final o engorde. En la etapa de cultivo intermedio, las semillas se seleccionaron por tallas y se colocaron en el interior de los pearl-nets a una densidad de 150 ejemplares/pearl-net. En la etapa de cultivo final o engorde estas fueron traspasadas a linternas ( 3 primeros pisos) a una densidad de 50 ejemplares/piso. El diseño estuvo compuesto por tres réplicas de linternas y tres réplicas de pearl-nets. Biometría y mantenimiento de sistemas de cultivo

Mensualmente se midió la altura valvar (L) en milímetros y el peso total (PT) en gramos de los ejemplares. Así mismo se realizó el mantenimiento de la línea y cambio de sistemas de cultivo sucios por limpios, a fin de evitar la presencia de depredadores y organismos incrustantes, los que al desarrollarse en gran abundancia pueden afectar el cultivo de manera negativa, ya que desgastan, deterioran los materiales de cultivo y modifican el flujo de agua, dejando las conchas sin suministro de alimento, pudiendo afectar negativamente el crecimiento (Pacheco \& Gárate, 2005), así como causar alta mortalidad.

Se determinó la temperatura y oxígeno superficial de la zona de trabajo durante los periodos en que se realizaron los experimentos.

Estimación del crecimiento

Para estandarizar los estimados de crecimiento en longitud, se determinó la tasa de crecimiento instantaneo relativo (TCIR) (Ricker, 1975; Xiao et al., 2005):

$$
\mathrm{TCIR}=\left(\left(\operatorname{Ln} L_{2}-\operatorname{Ln} L_{1}\right) / t\right) \times 100
$$

Donde $L_{2}$ y $L_{1}$ son la talla final e inicial respectivamente, $t$ es el intervalo de tiempo (en días) entre 2 muestreos consecutivos.

La tasa absoluta de crecimiento y los parámetros de crecimiento $\mathrm{K}$ y $\mathrm{L} \infty$ se determinaron usando el método de Gulland \& Holt (1959):

$$
L_{2}-L_{1} /(\mathrm{t} 2-\mathrm{t} 1)=a-b \mathrm{~L}
$$

Donde $L_{1}$ y $L_{2}$ son las longitudes correspondientes a los tiempos $t_{2}$ y $t_{1}$ y $\mathrm{L}=\left(L_{1}+L_{2}\right) / 2$. Los parámetros $\mathrm{K}=-b$ y $\mathrm{L} \infty=-\mathrm{a} / \mathrm{b}$

Para determinar la variación del rendimiento en crecimiento $(\Phi)$, se utilizó la ecuación de Pauly \& Munro (1984):

$$
\Phi=\log \mathrm{K}+2 \log \mathrm{L} \infty
$$

El factor de condición o factor de condición de Fultón (FC) fue estimado usando la relación entre el peso y la longitud (Froese, 2006):

$$
\mathrm{FC}=100 \mathrm{PT} / \mathrm{L}^{3}
$$

Donde: PT y L son el peso total (g) y la altura valvar (mm).

Análisis de datos

En los experimentos se aplicó un análisis de covarianza (ANCOVA) y una prueba t para comparar las curvas de regresión longitud- peso y determinar diferencias estadísticas entre los grupos estudiados (Sokal \& Rohlf, 1981). Para tal efecto las ecuaciones de regresión se linealizaron usando logaritmos:

$$
\log (\mathrm{P})=\log (\mathrm{a})+\mathrm{b} * \log (\mathrm{L}) .
$$


Asimismo se realizó un analisis de varianza de una via (ANOVA) para estimar diferencias entre las medias del FC en ambos experimentos.

\section{Resultados y Discusión}

En la Playa "El carbón", Pucusana, durante el periodo Junio a Noviembre del 2000, el promedio de temperatura superficial del mar fue $15.4{ }^{\circ} \mathrm{C}$ y oxígeno $4 \mathrm{mg} \mathrm{L}^{-1}$. De Junio a Diciembre del $2001,15.2^{\circ} \mathrm{C}$ y $3.9 \mathrm{mg} \mathrm{L}^{-1}$ respectivamente.

En la Tabla 1 y Tabla 2 se muestran la longitud media, TCIR y tasa de crecimiento absoluto de concha de abanico, estimados para los 2 experimentos.

En el primer experimento (Bahía IndependenciaLaboratorio), la longitud media de los ejemplares producidos en laboratorio fue significantemente menor al inicio $(\mathrm{F}=5.814 ; \mathrm{P}<0.05)$ y mayor al final $(\mathrm{F}=12.669 ; \mathrm{P}<0.05)$, con respecto a los ejemplares de Bahía Independencia, debido a que las semillas de laboratorio presentaron una mayor tasa de crecimiento (8.3 $\mathrm{mm} \mathrm{mes}^{-1}$ ) (Tabla 1; Figura 1a).

En el segundo experimento (Samanco-Lagunillas), la longitud media de los ejemplares de Lagunillas fue menor al inicio $(\mathrm{F}=32.02 ; \mathrm{P}<0.05)$ y mayor al final $(\mathrm{F}=45.53 ; \mathrm{P}<0.05)$, con respecto a los ejemplares de Samanco, cuya tasa de crecimiento fue menor (7.4 $\mathrm{mm} \mathrm{mes}^{-1}$ ) (Tabla 2; Figura 2a).

Las tasas de crecimiento promedio, en ambos experimentos son menores a las estimadas por Cisneros \& Arguelles (1994), quienes determinaron una tasa promedio de crecimiento de $11.7 \mathrm{~mm} \mathrm{mes}^{-1}$, en un trabajo realizado en la misma zona de estudio (Playa "El Carbon", Pucusana), con ejemplares de concha de abanico obtenidos en laboratorio, durante los años 1992-93, periodo en que se registró un aumento de la temperatura del mar. Las menores tasas obtenidas en el presente estudio, indican que esta especie crece más rapidamente en periodos cálidos (Mendo \& Wolff, 2003; Tarazona et al., 2007).

Las TCIR y de crecimiento absoluto (dL/dt), fueron mayores en los primeros meses de los experimentos y disminuyeron progresivamente (Tabla 1; Figura 1b y Figura 2b). De acuerdo a Wolff (1985), esta tendencia creciente en las tasas de crecimiento hasta cierto tamaño y su posterior decrecimiento, ha sido observado tambien en otros pectínidos.

Los valores de $L \infty$ y $\Phi$, estimados para ambos experimentos, corroboran lo estimado anteriormente, mayor crecimiento en los ejemplares procedentes de laboratorio ( $\mathrm{L} \infty 82$ y $\Phi 4.363$ ) con respecto a los de Bahia Independencia. De igual modo para los

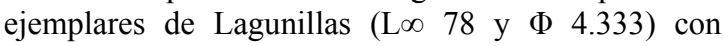
respecto a los de Samanco (Tabla 3). Los valores de $\Phi$ encontrados en este estudio son similares a los estimados durante eventos El Niño por Tarazona et al. (2007); Mendo \& Jurado (1993), lo cual demuestra que en cultivo suspendido se registran altos indices de $\Phi$ con respecto a ejemplares de ambiente natural, en condiciones ambientales normales, excepto los encontrados por Yamashiro \& Mendo (1988); Wolff (1985), durante El Niño 1982-83, quienes históricamente registran los mayores valores de $\Phi$ para esta especie, en ambiente natural.

Las pendientes de la relación longitud-peso, no presentaron diferencias significativas $(p>0.05)$ en los dos experimentos realizados. Sin embargo, si se observaron diferencias en los interceptos $(\mathrm{p}<0.05)$, cuyos valores fueron 0.000488 y 0.000481 para el grupo Bahía Independencia-Laboratorio, así como 0.000503 y 0.000463 para el grupo SamancoLagunillas (Tabla 5). Lo cual indica que existen diferencias en los grupos analizados, en relación a pesos totales para una misma talla.

Los ejemplares de Bahía Independencia presentaron mayor $\mathrm{FC}$, que los de laboratorio, para todos los rangos de tallas, excepto para el de 70-79 mm (Tabla 4, Figura 3a). Así mismo, los ejemplares de Lagunillas presentaron mayor FC que los de Samanco, sin embargo estas diferencias solo fueron observadas en los rangos 30-39 y 60-69 mm (Tabla 4, Figura 3b).

Los resultados muestran que conchas de abanico de diferentes poblaciones presentan diferencias en las tasas de crecimiento y FC. Existen varios factores ambientales como la temperatura y disponibilidad de alimento (Mendo \& Jurado, 1993; Mendo \& Wolff, 2003; Tarazona et al., 2007) que pueden determinar diferencias en el crecimiento y rendimiento en peso. Sin embargo, en este trabajo cada grupo estuvo sometido a las mismas condiciones de temperatura y oxígeno, por lo que las diferencias de crecimiento observadas, estarían dadas no por factores ambientales, sino por factores genéticos. En este sentido, dado que en bivalvos es usual encontrar una alta variabilidad genética en terminos de polimorfismo (de alelos) y heterocigosidad (Beaumont \& Beveridge, 1984), las diferencias en el crecimento y rendimiento entre ejemplares de Samanco y Lagunillas, pueden ser atribuidas a esta condición, tal como fue observado en el peso del músculo aductor de Argopecten purpuratus para diferentes poblaciones de Mejillones y Rinconada en Chile (Mogarat et al., 2001).

La heterocigosidad tambien está positivamente relacionada con la sobrevivencia y crecimiento de larvas y adultos (Fujio, 1982). Se ha observado que la heterocigosidad en poblaciones cultivadas es ligeramente más baja que en las poblaciones silvestres y reducida heterocigosidad ha sido encontrada en muchas especies de moluscos procedentes de laboratorio (Xiao et al., 2005). En este sentido, durante el presente estudio, los ejemplares de laboratorio presentaron mayor tasa de crecimiento, pero menor FC que los de Bahía Independencia, lo que pudo ser influenciado por lo antes mencionado. Sin embargo, no se puede precisar aún, que extención 
de reducción de la heterocigosidad contribuye a estas características (Xiao et al., 2005).

\section{Conclusiones}

Los resultados obtenidos nos permiten concluir que existen diferencias de crecimiento y factor de condición entre poblaciones de concha de abanico de diferentes procedencias. Es recomendable realizar análisis genéticos y otros estudios comparativos de crecimiento y supervivencia, en poblaciones silvestres y de laboratorio, con el fin de determinar los efectos de la variabilidad genética en los cultivos.

\section{Agradecimientos}

El presente trabajo se realizó con financiamiento del Instituto del Mar del Perú, en el marco del proyecto "Cultivo de Moluscos en Ambiente Natural". Se agradece a la empresa Mariexport S.A. por el apoyo logístico y facilidades brindadas para realizar el estudio en su concesión marina de Pucusana. Al Técnico Cristian Santos, de la Unidad de Investigaciones en Acuicultura del IMARPE, por su valiosa colaboración durante la ejecución de este trabajo.

\section{Literatura citada}

Alamo V. \& Valdivieso V. 1987. Lista sistemática de moluscos marinos del Perú. Boletín Volúmen Extraordinario Instituo del Mar del Perú.

Bandin R. \& Mendo J. 1999. Asentamiento larval de la concha de abanico (Argopecten purpuratus) en colectores artificiales en la Bahía Independencia, Pisco, Perú. Invest. Mar., Valparaíso. 27: 3-13.

Beaumont A.R. \& Beveridge C.M. 1984. Electrophoresis survey of genetic variation in Pecten maximus, Chlamys opercularis, C. varia and C. distorta from the Irish Sea. Marine Biology. 81: 299-306.

Cantillanez M., Avendaño M., Thouzeau G. \& Pennee M. 2005. Reproductive cycle of Argopecten purpuratus (Bivalvia : Pectinidae) in La Rinconada marine reserve (Antofagasta, Chile) : Response to environmental effects of E1 Niño and La Niña. Aquaculture. 246:181-195.

Cisneros R., Valdivieso V. \& Bautista J. 1992. Desarrollo larvario de Argopecten purpuratus L. Procedentes de reproductores parasitados. Memoria X Conabiol 02-07 agosto 1992, Lima-Perú. : 345-349.

Cisneros R. \& Arguelles J. 1994. Cultivo experimental de la concha de abanico Argopecten purpuratus (L.) a diferentes densidades y profundidades en sistema suspendido. La Acuicultura y el Desarrollo Sostenible, Santa Fé de Bogotá, Colombia. : 463-468.

De Franssu L. 1990. The worl market for bivalves - oyster mussel - clam - scallop. FAO/GLOBEFISH Research Program. Vol 4. : 115.

Di Salvo L.H., Alarcón E., Martínez E. \& Uribe E. 1984. Progress in mass culture of Chlamys (Argopecten) purpurata Lamarck (1819) with notes on its natural history. Rev Chil Hist Nat. 57: 35-45.

Froese R. 2006. Cube law, condition factor and weightlength relationship: history, meta-analysis and recommendations. J.Appl. Ichthyol. 22: 241-253.
Fujio Y. 1982. A correlation of heterozygosity with growth rate in the Pacific oyster, Crassostrea gigas. Tohoku J Agric Res. 33: 66-75.

Gulland J.A. \& Holt S.J. 1959. Estimation of growth parameters for data and unequal time intervals. J. Cons. Int. Explor. Mer. 25 (1) : 47-49.

Mendo J., Valdivieso V. \& Yamashiro C. 1988. Cambios en densidad, número y biomasa de la población de concha de abanico (Argopecten purpuratus) en la Bahía Independencia (Pisco, Perú) durante 1984-87. Bol. Inst. Mar Perú-Callao, Vol. Extraordinario. : 1-382.

Mendo J. \& Jurado E. 1993. Length-based growth parameter estimates of the Peruvian scallop (Argopecten purpuratus). Fisheries Research. 15: 357-367.

Mendo J., Isla L., Orrego H. \& Tomaylla R. 2001. Manual técnico para el cultivo y manejo integral de la concha de abanico, 74 pp. Programa APGEP-SENREM. Convenio USAID-CONAM. 1ra. Edición, Lima.

Mendo J. \& Wolff M. 2003. El impacto de El Niño sobre la producción de concha de abanico (Argopecten purpuratus) en Bahía Independencia, Pisco, Perú. Ecología aplicada. 2(1): 51-57.

Moragat D., Avendaño M., Peña J., Le Pennect M., Tanguyt A. \& Baron J. 2001. Genetic and morfological differentiation between two pectinid populations of Argopecten purpuratus from the northern chilean coast. Estud. Oceanol. 20: 51-60.

Pauly D. \& Munro J.L. 1984. Once more on growth comparison in fish and invertebrates. Fishbyte. 2: 21.

Pacheco A. \& Garate A. 2005. Bioincrustantes en estructuras de cultivo de Argopecten purpuratus en Bahía Samanco, Perú. Ecología Aplicada. 4(1,2): 149152.

Pacheco A. \& Stotz W. B. 2006. Will providing a filamentous substratum in the water column and shell litter on the bottom increase settlement and post-larval survival of the scallop Argopecten purpuratus? . Journal of Experimental Marine Biology and Ecology. 333:2739.

Ricker W.E. 1975. Computation and interpretation of biological statisties of fish population. Bulletin of the fisheries research borrad of Canada.

Sokal R. \& Rholf F. 1981. Biometry, 2nd edition, 859 pp. W.H. Freeman and Company, San Francisco.

Tarazona J., Espinoza R., Solis M. \& Arntz W. 2007. Crecimiento y producción somática de la concha de abanico (Argopecten purpuratus) en Bahía Independencia, Pisco (Perú) comparados entre eventos El Niño y La Niña. Revista de Biología Marina y Oceanografía. 42(3): 275-285.

Uddin M.J., Park K., Kang D., Park Y. \& Choi K. 2007. Comparative reproductive biology of Yeso scallop, Patinopecten yessoensis, under two different culture systems on the east coast of Korea. Aquaculture. 265:139-147.

Wolff M. 1985. Abundancia masiva y crecimiento de preadultos de la concha de abanico peruana (Argopecten purpuratus) en la zona de Pisco bajo condiciones de El Niño 1983. Bol. Inst. Mar Perú-Callao, Vol. Extraordinario. : 1-222.

Wolff M. 1988. Spawning and recruitment in the Peruvian scallop Argopecten purpuratus. Mar. Ecol. Prog. Ser. 42: 213-217. 
Wolff M., Taylor M., Mendo J. \& Yamashiro C. 2007. A catch forecast model for the Peruvian scallop (Argopecten purpuratus) based on estimators of spawning and settlement rate, Ecol. Model. 209(2-4): 333-341.

Xiao J., Ford S., Yang H., Zhang F. \& Guo X. 2005. Studies on mass summer mortality of cultured zhikong scallops (Chlamys farreri Jones et Preston) in China. Aquaculture. 250: 602-615.
Yamashiro C. \& Mendo J. 1988. Crecimiento de la concha de abanico (Argopecten purpuratus) en la Bahía Independencia, Pisco, Perú. Bol. Inst. Mar Perú-Callao, Vol. Extraordinario. : 1-382.

Yamashiro C., Rubio J., Taipe A. \& Aguilar S. 2002. Memorias I Jornada Científica Reserva Nacional Paracas. En : Mendo J. \& Wolf M. (Eds), pp. 77-87. Universidad Nacional Agraria La Molina.

\section{Tablas y figuras citadas en el texto}

Tabla 1. Longitud media (mm) +/- DS, tasa de crecimiento instantáneo relativo (TCIR) y tasa de crecimiento absoluta (dL/dt), de Argopecten purpuratus en cultivo suspendido en la Playa "El Carbón", Pucusana, con semillas de Bahía Independencia y Laboratorio (IMARPE).

\begin{tabular}{ccccc|cccc}
\hline & \multicolumn{3}{c|}{ Bahía Independencia } & \multicolumn{3}{c}{ IMARPE } \\
\hline Fecha & $\begin{array}{c}\text { LM } \\
(\mathrm{mm})\end{array}$ & DS & $\begin{array}{c}\text { TCIR } \\
\left(\mathrm{mm} \mathrm{dia}^{-1}\right)\end{array}$ & $\begin{array}{c}\mathrm{dL} / \mathrm{dt} \\
\left(\mathrm{mm} \mathrm{mes}^{-1}\right)\end{array}$ & $\begin{array}{c}\text { LM } \\
(\mathrm{mm})\end{array}$ & DS & $\begin{array}{c}\text { TCIR } \\
\left(\mathrm{mm} \mathrm{dia}^{-1}\right)\end{array}$ & $\begin{array}{c}\mathrm{dL} / \mathrm{dt} \\
\left(\mathrm{mm} \mathrm{mes}^{-1}\right)\end{array}$ \\
\hline 09.06 .00 & 23.5 & 3.1 & & & 22.8 & 1.9 & & \\
06.07 .00 & 29.1 & 4.2 & 0.784 & 6.2 & 30.7 & 2.8 & 1.101 & 8.8 \\
04.08 .00 & 39.9 & 7.2 & 1.088 & 11.2 & 42.3 & 4.7 & 1.104 & 12.0 \\
15.09 .00 & 54.9 & 4.2 & 0.758 & 10.7 & 57.2 & 4.6 & 0.716 & 10.6 \\
18.10 .00 & 61.9 & 5.5 & 0.367 & 6.6 & 65.0 & 4.2 & 0.386 & 6.7 \\
21.11 .01 & 65.5 & 5.1 & 0.167 & 3.3 & 67.9 & 5.2 & 0.133 & 3.2 \\
\hline Promedio & \multicolumn{7}{c}{7.6} \\
\hline
\end{tabular}

Tabla 2. Longitud media (mm) +/- DS, tasa de crecimiento instantáneo relativo (TCIR) y tasa de crecimiento absoluta (dL/dt), de Argopecten purpuratus en cultivo suspendido en la Playa "El Carbón", Pucusana, con semillas de Samanco y Lagunillas.

\begin{tabular}{|c|c|c|c|c|c|c|c|c|}
\hline \multirow[b]{2}{*}{ FECHA } & \multicolumn{4}{|c|}{ Samanco } & \multicolumn{4}{|c|}{ Lagunillas } \\
\hline & $\begin{array}{c}\text { LM } \\
(\mathrm{mm})\end{array}$ & DS & $\begin{array}{c}\text { TCIR } \\
\left(\mathrm{mm} \mathrm{dia}^{-1}\right)\end{array}$ & $\begin{array}{c}\mathrm{dL} / \mathrm{dt} \\
\left(\mathrm{mm} \mathrm{mes}^{-1}\right)\end{array}$ & $\begin{array}{l}\text { LM } \\
(\mathrm{mm})\end{array}$ & DS & $\begin{array}{c}\text { TCIR } \\
\left(\mathrm{mm} \mathrm{dia}^{-1}\right)\end{array}$ & $\begin{array}{c}\mathrm{dL} / \mathrm{dt} \\
\left(\mathrm{mm} \mathrm{mes}^{-1}\right)\end{array}$ \\
\hline 13.06 .2001 & 18.8 & 1.0 & & & 18.1 & 1.3 & & \\
\hline 06.07 .2001 & 24.4 & 2.1 & 1.127 & 7.3 & 24.9 & 2.1 & 1.393 & 8.9 \\
\hline 07.08 .2001 & 33.1 & 3.5 & 0.952 & 8.2 & 34.3 & 2.1 & 1.001 & 8.8 \\
\hline 11.09 .2001 & 46.3 & 3.5 & 0.962 & 11.3 & 47.7 & 2.6 & 0.945 & 11.5 \\
\hline 11.10 .2001 & 53.6 & 3.3 & 0.486 & 7.3 & 53.9 & 2.8 & 0.405 & 6.2 \\
\hline 12.11 .2001 & 58.7 & 3.2 & 0.284 & 4.8 & 62.0 & 2.5 & 0.440 & 7.6 \\
\hline 11.12 .2001 & 63.0 & 2.4 & 0.245 & 4.5 & 65.6 & 2.1 & 0.193 & 3.7 \\
\hline Promedio & & & & 7.4 & & & & 7.9 \\
\hline
\end{tabular}

Tabla 3. Parámetros de crecimiento de Argopecten purpuratus por periodo de estudio y grupos experimentales.

\begin{tabular}{lcccc}
\hline $\begin{array}{l}\text { Periodo de } \\
\text { estudio }\end{array}$ & Grupo & $k$ & L & $\Phi$ \\
\hline $\begin{array}{l}\text { Jun 2000- } \\
\text { Nov 2001 }\end{array}$ & B. Independencia & 3.17 & 81 & 4.318 \\
Jun 2000- & IMARPE & 3.43 & 82 & 4.363 \\
$\begin{array}{l}\text { Nov 2001 } \\
\text { Jun 2001- }\end{array}$ & Samanco & 4.08 & 72 & 4.325 \\
$\begin{array}{l}\text { Dic 2001 } \\
\text { Jun 2001- }\end{array}$ & Lagunillas & 3.54 & 78 & 4.333 \\
Dic 2001 & & & & \\
\hline
\end{tabular}

$\Phi=\log \mathrm{K}+2 \log \mathrm{L} \infty$
Tabla 4. Factor de condición (FC) por rango de tallas para Argopecten purpuratus en cada grupo experimental.

\begin{tabular}{|c|c|c|c|c|c|c|}
\hline \multirow{2}{*}{$\begin{array}{l}\text { Rango de tallas } \\
\mathrm{mm}\end{array}$} & \multicolumn{2}{|c|}{$\mathrm{N}^{\circ}$ de datos } & \multicolumn{2}{|c|}{ FC } & \multicolumn{2}{|c|}{ Anova } \\
\hline & \multicolumn{2}{|c|}{ B. Indep.lmarpe } & B. Indep. & Imarpe & $\mathrm{F}$ & $P$ \\
\hline $20-29$ & 231 & 194 & 23.0 & 21.6 & 37.07 & $<0.05$ \\
\hline $30-39$ & 121 & 120 & 20.8 & 20.0 & 8.37 & $<0.05$ \\
\hline $40-49$ & & 120 & 19.0 & 18.2 & 13.02 & $<0.05$ \\
\hline $50-59$ & 59 & 101 & 19.6 & 17.6 & 36.50 & $<0.05$ \\
\hline $60-69$ & 40 & 132 & 18.4 & 17.8 & 5.36 & $<0.05$ \\
\hline $70-79$ & 4 & 16 & 18.3 & 17.9 & 0.42 & $>0.05$ \\
\hline \multicolumn{3}{|c|}{ Rango de tallas $\mathrm{N}^{\circ}$ de datos } & \multicolumn{2}{|c|}{$\mathrm{FC}$} & \multicolumn{2}{|c|}{ Anova } \\
\hline $\mathrm{mm}$ & Saman & agunillas & Samanco & Lagunillas & $\mathrm{F}$ & $\mathrm{P}$ \\
\hline $20-29$ & 169 & 145 & 23.3 & 23.4 & 0.13 & $>0.05$ \\
\hline $30-39$ & 160 & 197 & 20.8 & 21.8 & 19.83 & $<0.05$ \\
\hline $40-49$ & 128 & 138 & 16.1 & 16.4 & 0.76 & $>0.05$ \\
\hline $50-59$ & 231 & 203 & 18.7 & 18.9 & 0.50 & $>0.05$ \\
\hline $60-69$ & 107 & 210 & 19.5 & 20.2 & 12.75 & $<0.05$ \\
\hline
\end{tabular}


Tabla 5. ANCOVA y prueba $t$ de la relación longitud - peso para ejemplares de Argopecten purpuratus, grupo Bahía Independencia - IMARPE, Samanco- Lagunillas. $\mathrm{p}<0.05$.

\begin{tabular}{|c|c|c|c|c|c|c|c|c|c|}
\hline & & & \multicolumn{4}{|c|}{ Prueba $\mathbf{t}$} & \multicolumn{3}{|c|}{ ANCOVA } \\
\hline & \multicolumn{2}{|c|}{ Grupos } & \multicolumn{2}{|c|}{ Pendientes (b) } & \multicolumn{2}{|c|}{ Interceptos (a) } & \multicolumn{3}{|r|}{ Nivel } \\
\hline & B. Indep. & IMARPE & $\mathrm{t}$ tab & $\mathrm{t}$ calc & $\mathrm{t}$ tab & $\mathrm{t}$ calc & $\mathrm{F}$ & $\mathrm{p}$ & Significancia \\
\hline Pendiente (b) & 2.759955 & 2.745415 & 1.96 & 0.82 & & & 0.34 & 0.559975 & NS \\
\hline \multirow[t]{4}{*}{ Intercepto (a) } & 0.000488 & 0.000481 & & & 1.96 & 10.05 & 21.16 & 0.000005 & $* * *$ \\
\hline & & & \multicolumn{4}{|c|}{ Prueba t } & \multicolumn{3}{|c|}{ ANCOVA } \\
\hline & \multicolumn{2}{|c|}{ Grupos } & \multicolumn{2}{|c|}{ Pendientes (b) } & \multicolumn{2}{|c|}{ Interceptos (a) } & & & Nivel \\
\hline & Samanco & Lagunillas & $\mathrm{t}$ tab & t calc & $\mathrm{t}$ tab & $\mathrm{t}$ calc & $\mathrm{F}$ & $\mathrm{p}$ & Significancia \\
\hline Pendiente (b) & 2.743403 & 2.775413 & 1.96 & 1.31 & & & 1.79 & 0.181155 & NS \\
\hline Intercepto (a) & 0.000503 & 0.000463 & & & 1.96 & 4.22 & 17.37 & 0.000033 & $* * *$ \\
\hline
\end{tabular}

F, valor experimental de la prueba de Fisher t calc $<=$ t tabla (no se rechaza $\mathrm{Ho}$ )

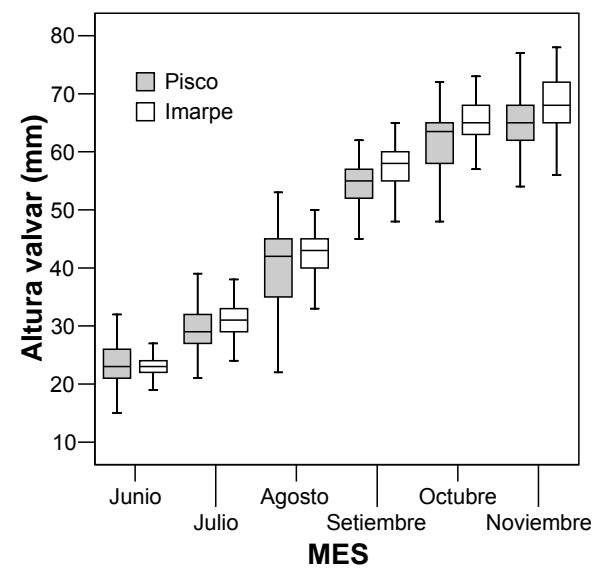

NS, no significativo

*** Altamente significativo

(a)

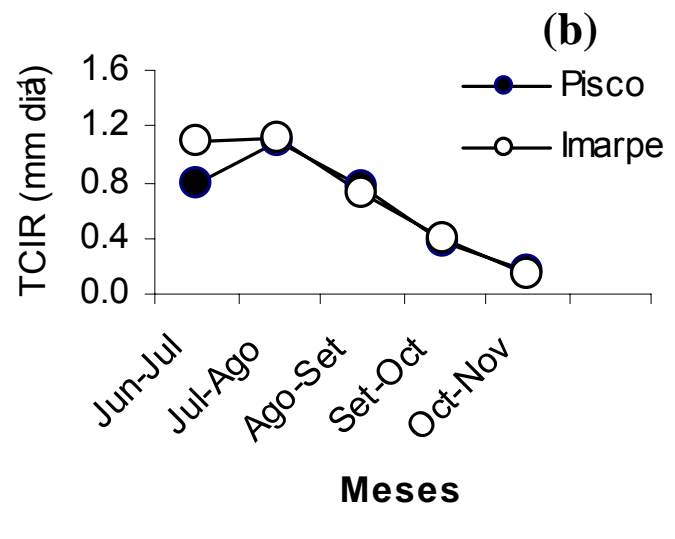

Figura 1. Crecimiento en longitud (altura valvar) (a) y tasa de crecimiento instantáneo relativo (TCIR) (b) de Argopecten purpuratus en cultivo suspendido, con semillas de Bahía Independencia (Pisco) y Laboratorio (IMARPE) en la Playa "El Carbón", Pucusana.

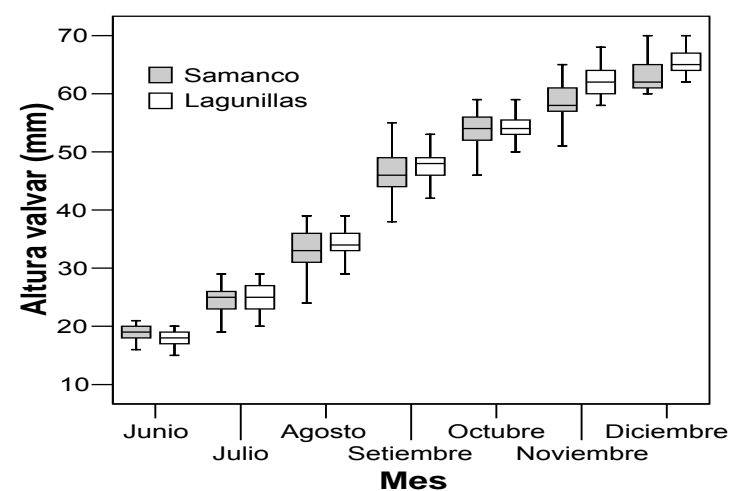

(a)

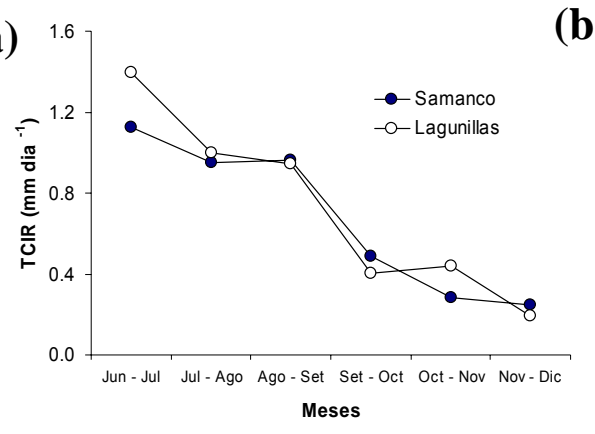

Figura 2. Crecimiento en longitud (altura valvar) (a) y tasa de crecimiento instantáneo relativo (TCIR) (b), de Argopecten purpuratus en cultivo suspendido, con semillas de Samanco y Lagunillas en la Playa "El Carbón", Pucusana. 


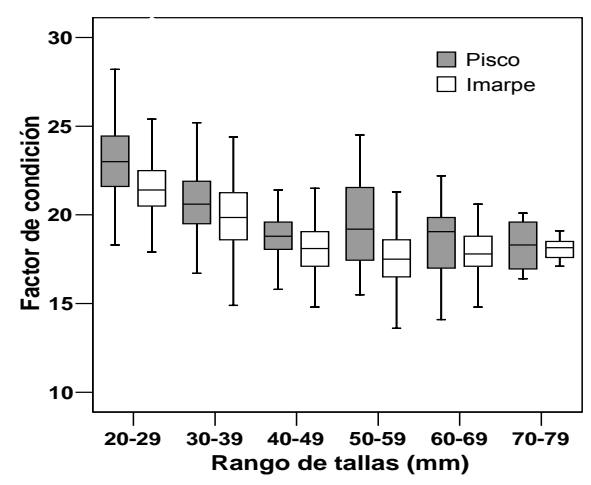

(a)

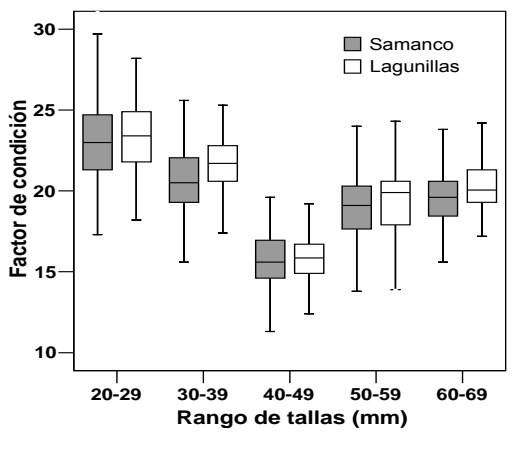

(b)

Figura 3. Factor de condición de Argopecten purpuratus en cultivo suspendido, grupo Bahía Independencia (Pisco)-Laboratorio (IMARPE) (a) y Samanco-Lagunillas (b) en la Playa "El Carbón", Pucusana.

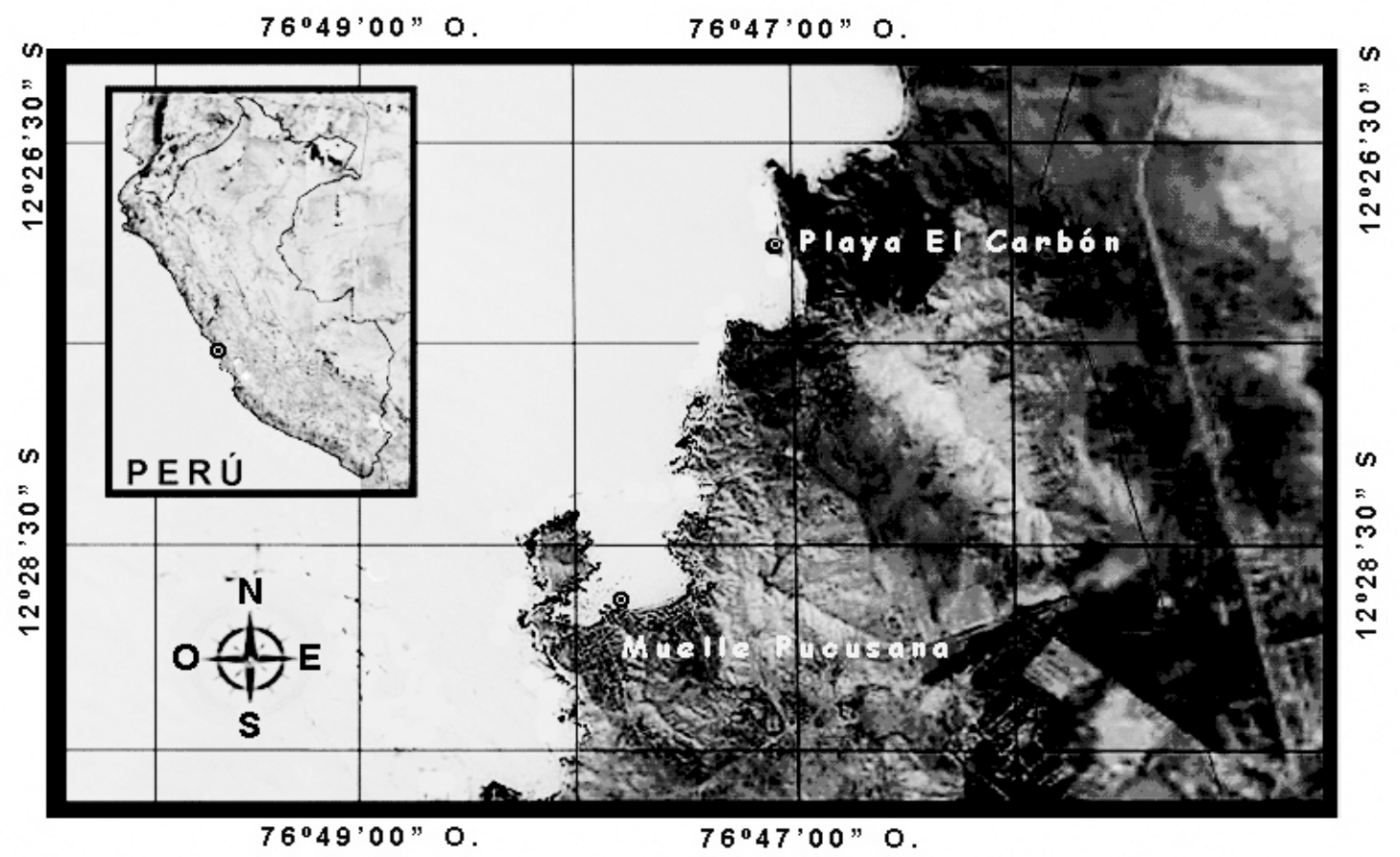

Figura 4. Mapa de la Playa “El Carbón”, Pucusana (Cortesía Blgo. Carlos Vergara).

${ }^{1}$ Unidad de Investigaciones en Acuicultura. Instituto del Mar del Perú. Ap. 22, Chucuito-Callao, Perú. rcisneros@imarpe.gob.pe

${ }^{2}$ Unidad de Investigaciones en Invertebrados Marinos. Instituto del Mar del Perú. Ap. 22, Chucuito-Callao, Perú. 